\title{
Comparacion entre Valores de Vtec Predichos por el Modelo Nequick2 y los Obtenidos a Partir de Gps sobre Boulder durante muy baja actividad Solar
}

\author{
Comparison between Vtec Values Predicted by the \\ Nequick2 Model and those Obtained from Gps on \\ Boulder during Very Low Solar Activity
}

Presentación: 02/05/2018

Aprobación: 21/08/2018

\section{Joaquín Marcelo Lopez}

CIASUR, Facultad Regional Tucumán - Universidad Tecnológica Nacional- Argentina joaquinmarcelolopez@gmail.com

\section{Rodolfo Gerardo Ezquer}

FACET, CIASUR, Universidad Nacional de Tucumán - Universidad Tecnológica Nacional Consejo Nacional de Investigaciones Científicas y Técnicas - Argentina

rezquer@herrera.unt.edu.ar

\section{Marcelo Roque Albornoz}

CIASUR, Facultad Regional Tucumán - Universidad Tecnológica Nacional - Argentina marceloalb@gmail.com

\section{Carlos Exequiel Garay}

CIASUR, Facultad Regional Tucumán - Universidad Tecnológica Nacional - Argentina exequielgaray@gmail.com

\section{Luis Alberto Scidá}

CIASUR, FACET, Facultad Regional Tucumán - Universidad Tecnológica Nacional - Argentina Iscida@herrera.utn.edu.ar 


\title{
Resumen
}

Una especificación confiable del estado de la ionósfera dada por modelos empíricos es importante para mitigar los efectos que esta zona atmosférica produce sobre las operaciones de los sistemas de posicionamiento y navegación basadas en satélites.

Este estudio evalúa la capacidad del modelo NeQuick2 para predecir el contenido electrónico vertical total (VTEC), hasta la altura de los satélites GPS, sobre Boulder $\left(40,00^{\circ} \mathrm{N}, 254,75^{\circ} \mathrm{E}\right)$, durante una época de muy baja actividad solar. Para ello se comparan las predicciones del modelo con valores de VTEC obtenidos a partir de señales de satélites GPS, publicados por el National Geophysical Data Center (NGDC) de EEUU.

Teniendo en cuenta las incertezas de los valores de GPS VTEC publicados por el NGDC, los resultados muestran que el modelo estudiado, en general, se comporta como un buen predictor del contenido electrónico total en las condiciones solares consideradas.

Palabras claves: Ionosfera; contenido electrónico tota; GPS; vtec; Modelo NeQuick2; Latitud media.

\begin{abstract}
A reliable specification of the state of the ionosphere given by empirical models is important to mitigate the effects that this ionospheric area produces on the operations of positional and navigation systems based on satellites.

This study evaluates the ability of the NeQuick2 model to predict vertical, total electron content (VTEC), to the height of the GPS satellites, over Boulder $\left(40,00^{\circ} \mathrm{N}\right.$, $254.75^{\circ} \mathrm{E}$ ), during a time of very low solar activity. To do this the model predictions are compared with VTEC values obtained from GPS satellite signals, published by the National Geophysical Data Center (NGDC) U.S.

Taking into account the uncertainties of the values of GPS VTEC published by the NGDC, the results show that this model generally behaves as a good predictor of the total electron content in the solar conditions considered.
\end{abstract}

Keywords: Ionosphere; Vertical electron content; GPS; vtec; NeQuick2 model; middle latitude

\section{Introducción}

La zona de la atmosfera que va desde los $50 \mathrm{~km}$ hasta $1052000 \mathrm{~km}$ de altura, presenta ionización suficiente como para afectar las de ondas de radio que a través de ella se propagan. Por esto se la designa como Ionósfera. La ionización presente en la ionósfera es producida principalmente por radiación ultravioleta y rayos $\mathrm{X}$ 
solares.

La cantidad de electrones libres en un cilindro con una sección transversal de $1 \mathrm{~m} 2$, que se extiende a lo largo del camino de una señal que viaja atravesando la ionósfera desde un punto A (por ejemplo, un satélite en órbita) hasta un punto B (un receptor de señal satelital en tierra) se denomina Contenido Electrónico Total (en inglés, Total Electron Content - TEC), y es una magnitud muy útil a la hora de analizar como la ionósfera afecta la propagación de las ondas de radio.

La expresión formal para definir el TEC es la siguiente:

$$
\operatorname{TEC}=\int_{A}^{a} \mathrm{~N} d \mathrm{l}
$$

donde $\mathrm{N}$ es la densidad electrónica (en $\mathrm{m}$-3), dl es el incremento en distancia (en m) y la integral se calcula a lo largo del camino que une los puntos A y B.

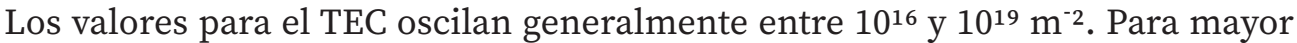
simplicidad, el TEC suele expresarse en la Unidad de Contenido Electrónico Total (en inglés, Total Electron Content Unit - TECU), con la siguiente equivalencia:

$$
1 \mathrm{TECU}=10^{16} \mathrm{~m}^{-2}
$$

El TEC varía dependiendo de la hora del día, la estación de año, la actividad solar (ciclo solar), la actividad magnética, la latitud geomagnética, etc. Además, se observa una importante variación de un día para otro, lo que hace muy difícil su predicción.

\section{El GPS}

El sistema de posicionamiento global (Global Positioning System - GPS) fue desarrollado por el Departamento de Defensa de los Estados Unidos como un sistema de navegación y difusión de la escala de tiempo. Este sistema consta de tres elementos principales: a) un conjunto de satélites que emiten señales electromagnéticas, b) los receptores que reciben dichas señales y c) una serie de estaciones de control. A partir de las señales recibidas de al menos cuatro satélites simultáneamente y conocida la posición de éstos es posible estimar ( $\mathrm{x}, \mathrm{y}, \mathrm{z}, \mathrm{t})$ para el receptor, esto es, su ubicación en un sistema de coordenadas geográfico mundial en tiempo real.

El GPS por lo tanto es un sistema pasivo, ya que no necesita interactuar con cada receptor, sino que es este último el que, simplemente recibiendo la señal, es capaz de realizar todos los cálculos de manera independiente. Esta unidireccionalidad hace que estos sistemas sean accesibles a un número ilimitado de usuarios. 
El GPS funciona mediante una red de 24 satélites en órbita sobre el planeta Tierra, a $20200 \mathrm{~km}$ de altura, con trayectorias sincronizadas para cubrir toda la superficie de la Tierra. Cuando se desea determinar la posición, el receptor que se utiliza para ello localiza automáticamente como mínimo tres satélites de la red, de los que recibe unas señales indicando la identificación y la hora del reloj de cada uno de ellos.

El sistema GPS es, en esencia, un sistema de medida de tiempos, donde el observable básico es el tiempo de retardo entre la emisión de la señal por el satélite y la llegada a nuestro receptor. Cada satélite GPS transmite señales centradas en dos frecuencias de radio de microondas, $1575.42 \mathrm{MHz}$, llamada Link1 o L1, y 1227.60 $\mathrm{MHz}$, Link2 o L2. Estos canales pertenecen a la banda de frecuencias L (1-2 GHz). Ambas son múltiplos de la frecuencia fundamental 10,23 MHz, patrón de tiempo del sistema.

Si los relojes con los que medimos el tiempo mencionado, correspondiente a un satélite, estuvieran perfectamente sincronizados y las señales se propagaran en el vacío, este tiempo de retardo multiplicado por la velocidad de la luz en el vacío, c, nos estaría midiendo $\rho$, que es la distancia geométrica al satélite en cuestión, también llamada Rango.

Sin embargo, en la práctica la situación dista bastante de la ideal mencionada, ya que en el proceso de medición se presentan errores atribuibles a: falta de sincronización entre los relojes del satélite y los del receptor, retardos en la propagación producidos por la ionósfera y la Troposfera, retardos en el hardware de los dispositivos, recepción multicamino, ruido.

Luego, la distancia satélite-receptor medida, afectada por los factores mencionados, es $R_{P}$, y se denomina Pseudorango, el que puede expresarse como:

\section{$R_{P}=\rho+c\left(d t_{r}-d t^{s}\right)+T+$

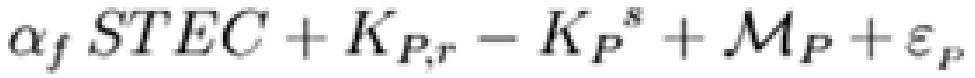

en la que, $\rho$ es el rango, el cual representa la distancia geométrica entre el satélite y el receptor, $c\left(d t_{r}-d t^{s}\right)$ representa el error debido a los desfasajes entre los

relojes del satélite y del receptor, incluyéndose efectos relativistas, $T$ es el error causado por el retardo Troposférico en la propagación de la señal. $K_{P, r}-K_{P}{ }^{s}$ es el error debido a retardos en el instrumental del satélite y el receptor, $\mathcal{M}_{P}$ representa el efecto generado por la recepción de señales multitrayecto, dependiente de la frecuencia y código usado en la señal, ${ }^{\varepsilon_{P}}$ es el error producido por el ruido en el receptor, $\alpha_{f} S T E C$ y es el error introducido por la ionósfera, el cual es dependiente de la frecuencia de la señal. En este término el factor $S T E C$ es el TEC calculado a 
lo largo del camino de la señal desde el satélite en cuestión y el receptor, expresado en unidades TECU mientras que $\alpha_{f}$ es un factor de conversión definido como:

$$
\alpha_{f}=\frac{40.3}{f^{2}} 10^{16}
$$

cuyas unidades son

[m / TECU] y donde el valor de $\mathrm{f}$ debe ser el que corresponde a la frecuencia de la señal en Hz.

\section{Receptores GPS Doble Frecuencia}

Utilizando un receptor GPS de doble frecuencia (recepciona simultáneamente las señales L1 y L2) se puede medir el Pseudorango correspondiente a cada frecuencia, siendo los mismos RP1 para la señal de Link1 y RP2 para la señal de Link2.

Estos pueden combinarse para definir un Pseudorango RPC, de la forma

$$
R_{P C}=\frac{f_{1}^{2} R_{P 1}-f_{2}^{2} R_{P 2}}{f_{1}^{2}-f_{2}^{2}}
$$

el cual esta libre del error que introduce la ionósfera .

Otra combinación posible de los Pseudorangos es

$$
R_{P I}=R_{P 2}-R_{P 1}
$$

en el que se cancela la parte geométrica de las mediciónes, quedando solo los efectos dependientes de la frecuencia (entre ellos el retardo ionosferico).Justamnte esta combinacion, conocida como "Geometry-free", puede utilizarse para estimar el STEC.

Receptores GPS Simple Frecuencia:

Con receptores GPS de simple frecuencia (solo pueden recepcionar una de las señales de los satélites, que son los más económicos y usados por ejemplo en navegación) solo se cuenta

con la medición del Pseudorango RP1.Debido a esto, como no es posible estimar directamente el error introducido por la ionósfera, se hace necesario su modelado 
para luego proceder a la corrección de la medición del rango.

Validación de Modelos Ionosfericos con mediciones de TEC:

Con lo visto hasta aquí, queda clara la importancia de validar valores de TEC modelados comparándolos con valores basados en mediciones.

A pesar de que la mayoría de las mediciones de TEC corresponden a TEC Oblicuo (STEC) con ubicaciones arbitrarias de satélites respecto de los receptores, es posible el cálculo del VTEC correspondiente.

Este proceso, se basa en asumir a la ionósfera como una delgada capa a altura fija. Este método presenta sus limitaciones en cuanto a la exactitud de los valores de VTEC obtenido, presentando un error de algunos TECU. (Kersley et al, 2004).

Modelos, Datos y Comparación:

Los modelos ionosféricos empíricos tienen varias ventajas, entre ellas:

- Permiten calcular la densidad electrónica ionosférica en función de altura.

- Generan un "Perfil Ionosférico" para un dado lugar, en un dado instante, para un grado de actividad solar definido.

- Permiten por Integración Calcular VTEC (Contenido Electrónico Total Vertical) y el STEC (Contenido Electrónico Total Oblicuo).

- Permiten realizar corrección de efecto ionosférico en GPS de Simple Frecuencia.

\section{Modelo: NeQuick2}

NeQuick es un modelo ionosférico para la determinación de la densidad de electrones en la ionósfera, desarrollado en el Laboratorio de Aeronomía y Radiopropagación (actual Telecommunications/ ICT for Development Laboratory) del Abdus Salam International Centre for Theoretical Physics (ICTP), Trieste, Italia y en el Institute for Geophysics, Astrophysics and Meteorology de la Universidad de Graz, Austria, en el marco de un proyecto de la European Commission COST (Cooperation in the field of Scientific and Technical Research) Action 251.

Mejoras en este modelo fueron introducidas por Radicella y Leitinger (2001).

Una modificación del perfil de base fue introducida por Leitinger et al (2005).

Un perfil de tope modificado fue propuesto por Coïsson et al (2006).

Una nueva versión de este modelo fue presentada por Nava et al (2008).

Este modelo incluye seis capas semi-Epstein con puntos definidos por los valores de foE, foF1, foF2 y $\mathrm{M}(3000) \mathrm{F} 2$.

Los datos de entrada de este modelo son posición, tiempo y flujo solar y los datos 
que entrega a la salida son, la concentración de electrones en una dada localización y tiempo, así como su integración en altura, o sea, TEC.

\section{Datos: GPS VTEC del National Geophysical Data Center (NGDC)}

Los valores de VTEC sobre la zona continental de EEUU pueden verse con una evaluación en tiempo casi real en la página de internet:

http://www.ngdc.noaa.gov/stp/IONO/USTEC/products/.

Allí dicha información es publicada por el Centro Nacional de Datos Geofísicos (National Geophysical Data Center - NGDC) de la Administración Nacional Oceánica y Atmosférica (National Oceanic and Atmospheric Administration - NOAA).

La obtención del VTEC está basada en el uso de datos de receptores de señales de satélites GPS de doble frecuencias y utiliza un modelo de asimilación de datos.

En la base de datos de US VTEC, los datos están agrupados cada un grado en latitud y longitud formando una cuadrícula de dos dimensiones, con valores que se actualizan cada 15 minutos. La región cubierta se extiende desde $10^{\circ} \mathrm{N}$ hasta $60^{\circ} \mathrm{N}$ y desde $50^{\circ} \mathrm{O}$ hasta $150^{\circ} \mathrm{O}$.

Como la técnica de cálculo de VTEC consiste en obtenerlo como equivalente de STEC, los datos presentan una incerteza del orden de +/- 2TECU.

\section{Comparación:}

Para el presente trabajo se tomaron los valores de VTEC provistos por la NGDC para las coordenadas de Boulder, esto es $\left(40,00^{\circ} \mathrm{N}, 254,75^{\circ} \mathrm{E}\right)$, para los Años $2008 \mathrm{y}$ 2009, los cuales corresponden a ciclos de muy baja actividad solar.

Se utilizaron los datos horarios desde las 00 del tiempo universal (Universal Time - UT) a las 23 UT correspondiente a todos los días de Marzo (equinoccio), Junio (solsticio), Septiembre (equinoccio) y Diciembre (solsticio) de 2008 y 2009.

Se tomó como valor representativo de VTEC para cada hora de un mes en particular a la mediana del VTEC a esa hora, calculada sobre el conjunto de los días de ese mes.

En NeQuick2 se cargaron los parámetros correspondientes a la ubicación de Boulder para los mismos meses de los años 2008 y 2009.Se indicó que el parámetro R12 (el cual es una media corrida de 12 meses del número de manchas solares) fuera tomado de la base de datos de la NGDC.

Se hizo una corrida del programa para cada hora de todos los días de los meses bajo análisis, obteniéndose una serie de valores con el mismo formato al de los datos extraídos de la NGDC. Como altura de tope para el cálculo se puso $20200 \mathrm{~km}$ que es la altura de orbita de los satélites GPS.

Una vez completos los dos grupos de Valores (Datos NGDC y NeQuick2), se procedió a graficarlos en forma superpuesta para cada mes en cuestión.

En las gráficas, las barritas horizontales representan las cotas superior e inferior de incerteza de los datos de VTEC de NGDC.

A continuación, se muestran los resultados con una breve descripción: 
Variación horaria de VTEC de NeQuick2 y VTEC experimental marzo 2008 - marzo 2009
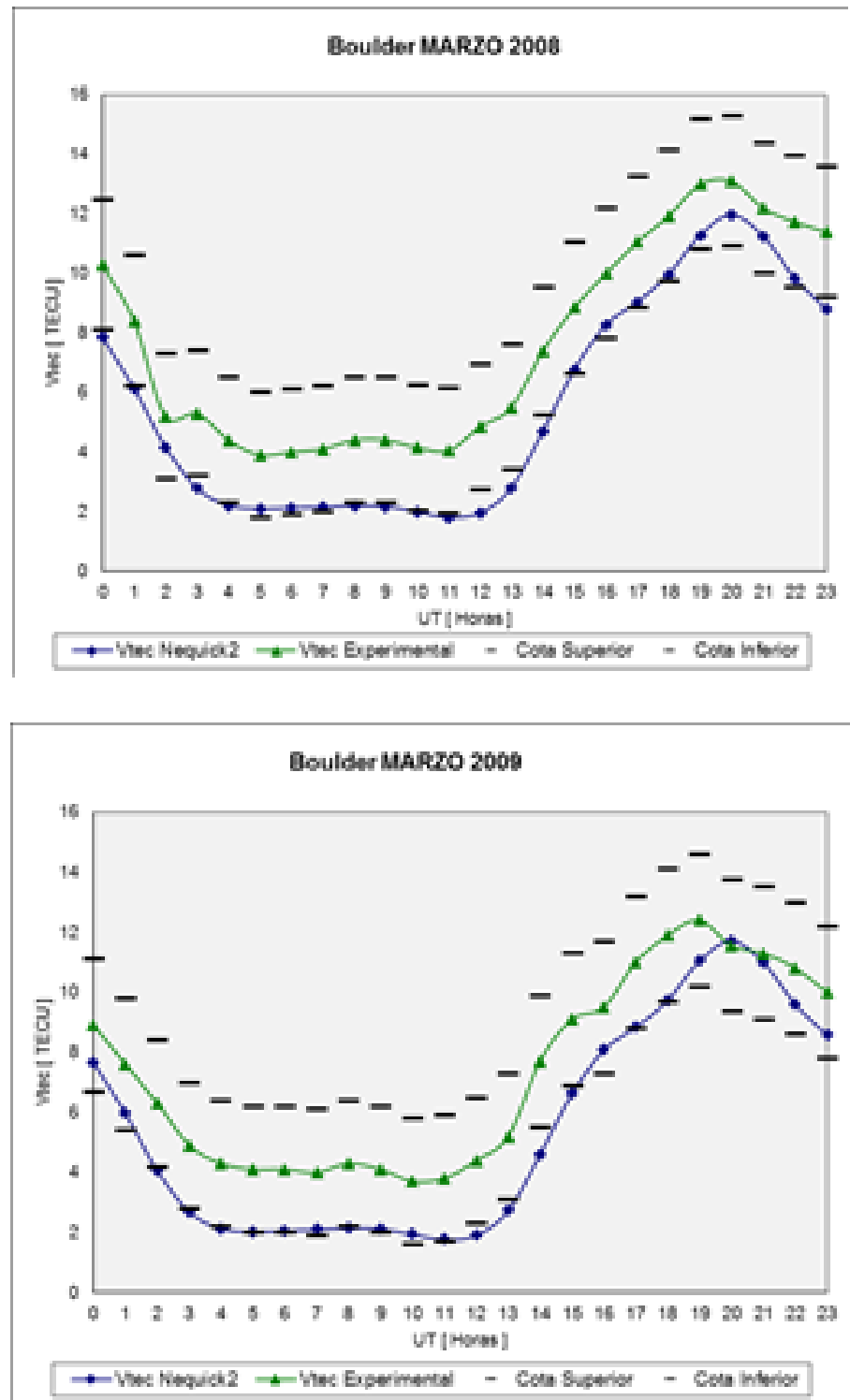

Figura 1: VTEC medido y modelado. Boulder Marzo 2008 y Marzo de 2009.

En la figura 1, se muestra la comparación de los valores de la mediana mensual del VTEC medido sobre Boulder con señales de satélites GPS y las predicciones de NeQuick2 correspondientes a Marzo de 2008 y Marzo de 2009.

Para Marzo de 2008, se observa que durante las horas nocturnas (las de menor ionización) los valores modelados son menores que los medidos en una cantidad aproximada de 2 TECU. Esta diferencia se hace menor en las horas de mayor 
ionización. Es decir que a pesar de que en Nequick2 se utilizó como altura límite de integración los 20200 km (altura de orbita de los satélites GPS), este subestima el valor calculado frente a los valores experimentales.

Para Marzo de 2009 se nota que de 0 UT a 19 UT, el valor modelado es menor al medido, entre las 20 UT y 21 UT el modelo coincide con lo medido. Luego entre las 22 UT y 23 UT se vuelve a tener la diferencia anterior.

Variación horaria de VTEC de NeQuick2 y VTEC experimental Junio 2008 - Junio 2009
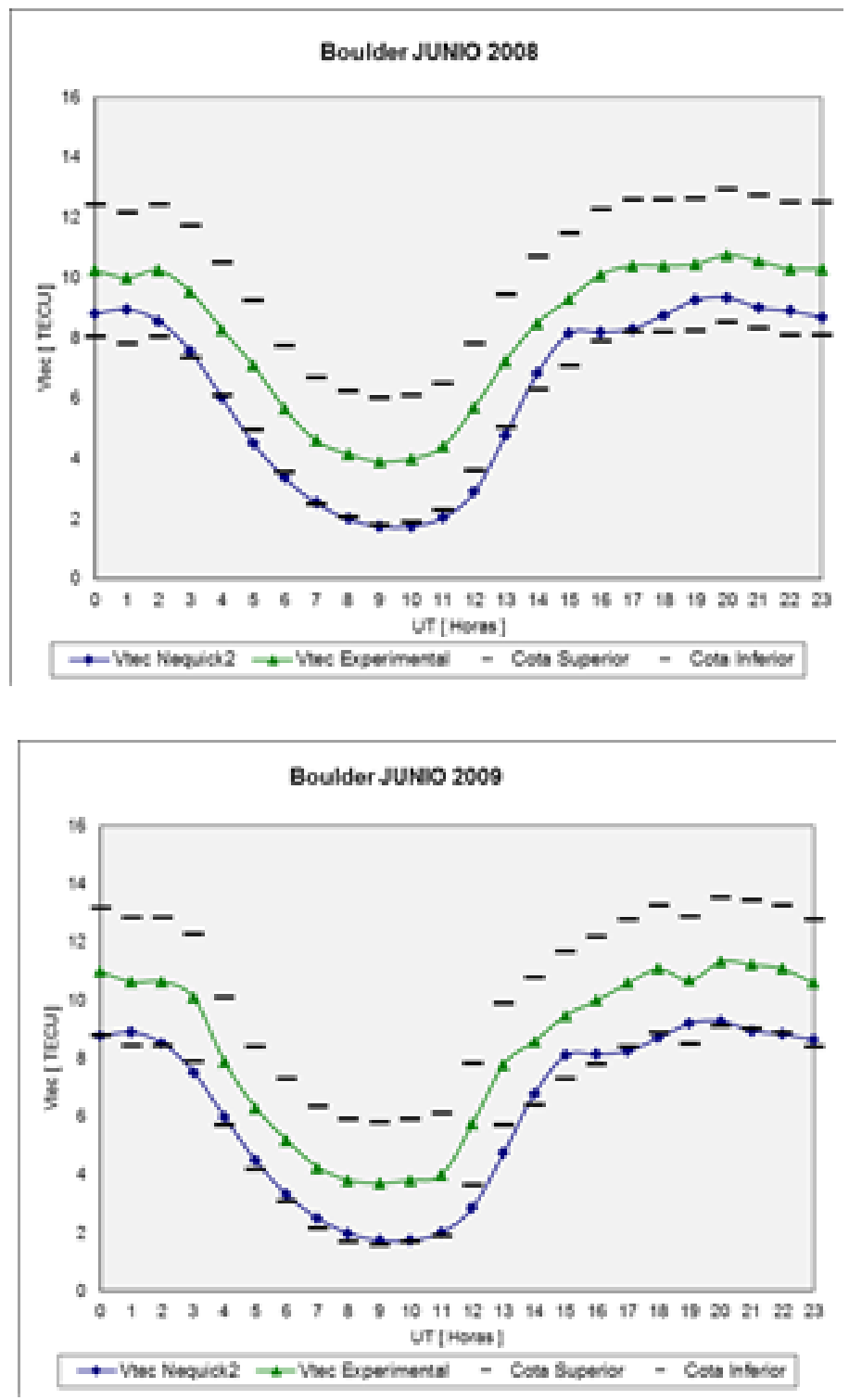

Figura 2: VTEC medido y modelado. Boulder Junio 2008 y Junio de 2009. 
En la figura 2 se presenta la comparación de las mismas magnitudes que en la figura 1, pero correspondientes a los meses de Junio de 2008 y Junio de 2009. Como se ve en estas gráficas el valor medido es mayor que el modelado, comportamiento similar al visto en los meses de Marzo.

Variación horaria de VTEC de NeQuick2 y VTEC experimental Septiembre 2008 - Septiembre 2009
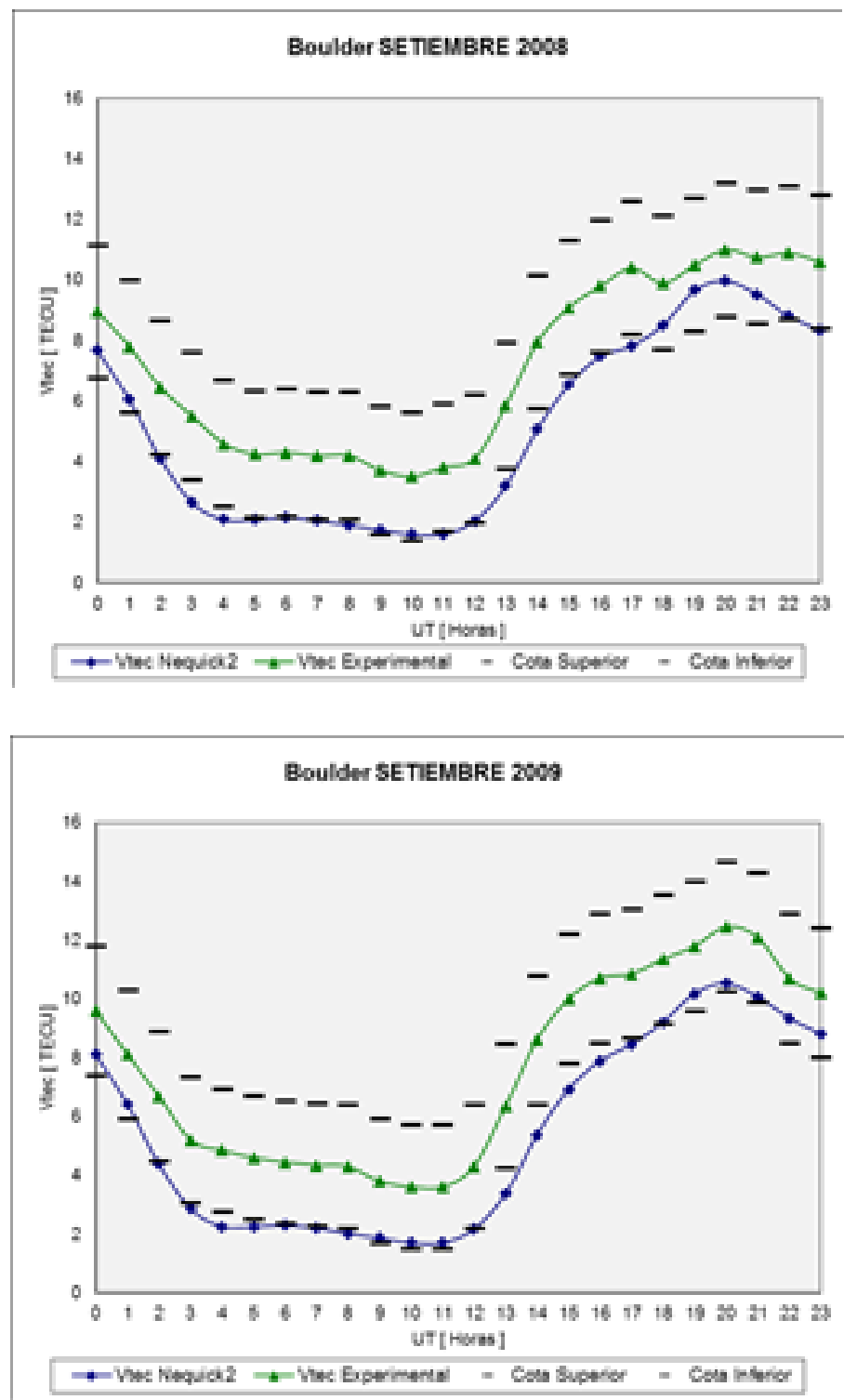

Figura 3: VTEC medido y modelado. Boulder Septiembre 2008 y Septiembre de 2009.

Como se puede apreciar en la figura 3 para el mes de Septiembre del año 2008, 
vemos que entre las 0 UT y las 17 UT el valor medido mantiene la diferencia con el valor modelado. Entre las 18 UT y las 21 UT se acercan bastante ambas curvas. Luego hasta el final entre las 22 UT y las 23 UT se observa una diferencia de 2 TECU aproximadamente.

En el mes de Septiembre de 2009 el valor medido y el valor modelado mantienen una diferencia constante, a lo largo de toda la curva.

Variación horaria de VTEC de NeQuick2 y VTEC experimental Diciembre 2008 Diciembre 2009
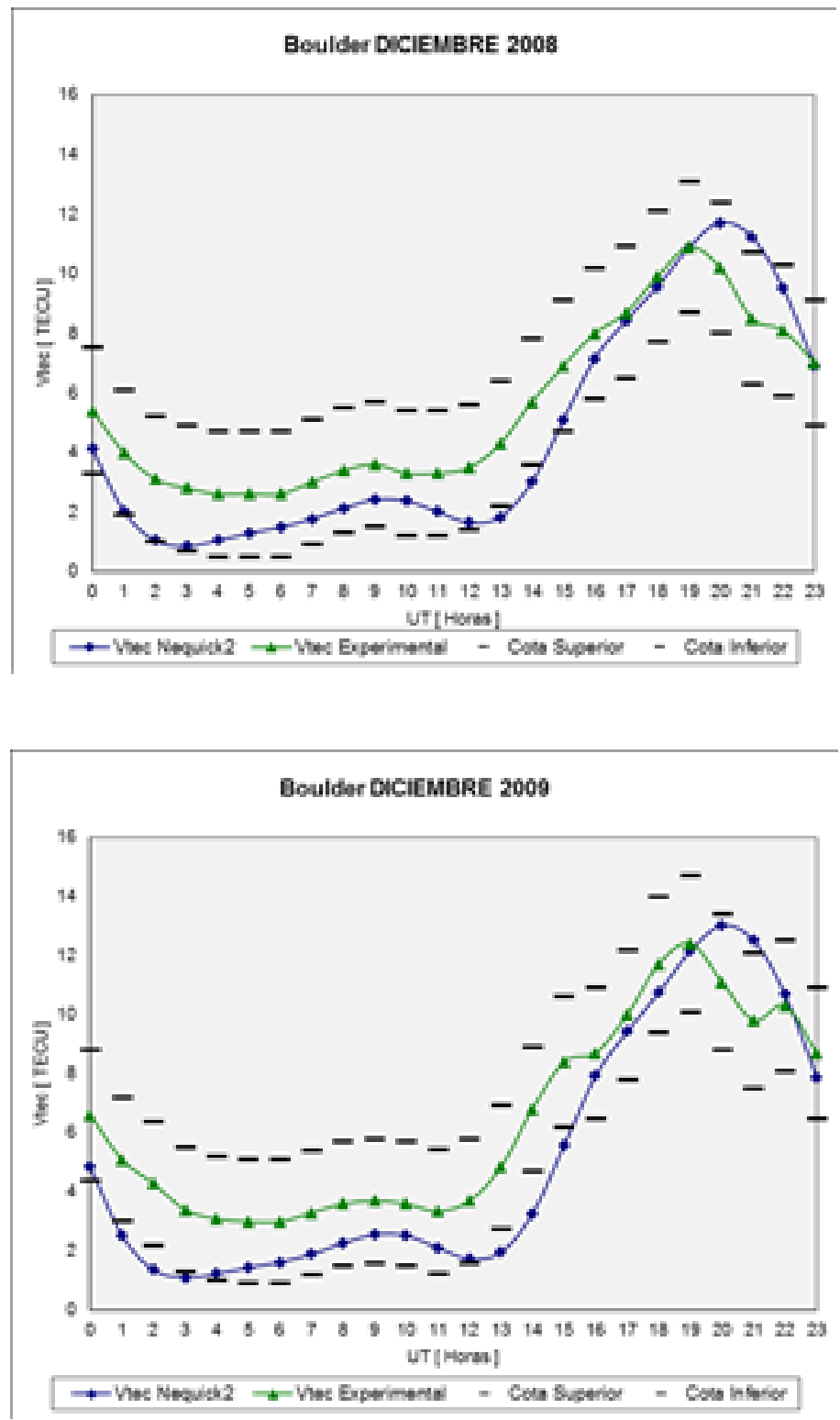

Figura 4: VTEC medido y modelado. Boulder Diciembre 2008 y Diciembre de 2009. 
En la figura 4 se observa que para el mes de Diciembre tanto de 2008 como de 2009, los valores medidos y modelados tienen coincidencias notables.

Para Diciembre de 2008 la diferencia se mantiene entre las 0 UT y las 15 UT. Entre las 16 UT y las 19 UT hay casi una coincidencia entre los valores medidos y los valores modelados para 2008. Entre las 20 UT y las 23 UT el valor modelado con NeQuick2 sobreestima al valor medido.

Para Diciembre de 2009 sucede algo similar al año anterior. Entre las 0 UT y las 15 UT se mantiene la diferencia entre el valor medido y el valor modelado. Entre las 16 UT y las 19 UT hay casi una coincidencia entre los valores medidos y los valores modelados. Entre las 20 UT y las 22 UT el valor modelado con NeQuick2 sobreestima al valor medido.

$\mathrm{Y}$ a las 23 UT el valor modelado vuelve a ser un poco menor que el valor medido.

La excepción la constituye Diciembre en horas de máxima ionización en donde se ve que el modelo sobrestima el VTEC ionosférico. Esto sugiere que, en general, NeQuick2 puede ser considerado como buen predictor del VTEC en latitudes medias Norte para el mínimo solar profundo ocurrido en 2008 y 2009.

\section{Conclusiones:}

Como se vio en estudios realizados anteriormente (Ezquer et al, 2014), el desempeño de los modelos depende de la zona geográfica que se considere, siendo peor para bajas latitudes. Para los meses representativos estudiados, (Marzo, Junio, Setiembre y Diciembre, de los años 2008 y 2009), el Modelo NeQuick2, en general presenta variaciones similares a las medidas con GPS a lo largo del día.

Teniendo en cuenta la incertidumbre de las mediciones con GPS, NeQuick2 arroja valores dentro de la franja de la medición. Es probable que NeQuick2 subestime valores por no presentar buen desempeño en el cálculo a alturas plasmasféricas. (Arriba de los $2000 \mathrm{~km}$ hasta los $20200 \mathrm{~km}$ ).

En general se observa un buen desempeño del modelo NeQuick2 para años de Baja Actividad Solar y Latitudes Medias. 


\section{Referencias}

https://gssc.esa.int/navipedia/index.php/GNSS_Basic_Observables

Ezquer R.G., Radicella,S.M. (2008): Ionósfera de bajas latitudes: efectos sobre señales de satélites recibidas en Tucumán, Física de la Tierra 61 2008, 20 61-81.

Hartmann G. K. and Leitinger R.,(1984): Range error due to ionospheric and tropospheric Effects for signals frequencies above 100 Mhz., Bull. Geod.58, 109136, 1984.

Kersley L. et al , 2004: Total electron content - A key parameterin propagation: measurement and use in ionospheric imaging. ANNALS OF GEOPHYSICS, SUPPLEMENT TO VOL. 47, N. 2/3, 2004.

Nava, B., P. Coisson and S.M. Radicella (2008): A new version of the NeQuick ionosphere electron density model, Journal of Atmospheric and Solar-Terrestrial Physics, doi:10.1016/j.jastp.2008.01.015.

Radicella, S.M., Leitinger, R., The evolution of the DGR approach to model electron density pro-files, Adv. Space Res., 27, 35-40, 2001.

Coïsson, P., S.M. Radicella, R. Leitinger, B. Nava (2006), Topside electron density in IRI and NeQuick: features and limitations , Advances in Space Research (37), 937-942.

Leitinger, R., Zhang, M. L. and Radicella, S. M. (2005), An improved bottomside for the ionospheric electron density model NeQuick, Annals of Geophysics, Volume 48, N. 3, 525-534, DOI: 10.4401/ag-3217.

Ezquer R.G., Garay C.E., Albornoz M.R., Cabrera M.A, Jadur C., Predicción del contenido electrónico ionosférico vertical sobre una estación de latitud media norte durante un minimo solar profundo. , Ciencia Tecnología y Medio Ambiente, Fac. Regional Tucumán, Universidad Tecnológica Nacional, vol 14, 57-64, 2014.

\section{Agradecimientos}

Agradecemos al Telecommunications/ICT for Development (T/ICT4D) Laboratory of the Abdus Salam International Centre for Theoretical Physics, Trieste, Italy por proveer NeQuick2 en su version online:

http://t-ict4d.ictp.it/nequick2/nequick-2-web-model.

Tambien agradecemos al National Geophysical Data Center (NGDC) de EEUU, por brindar acceso a los datos de VTEC sobre Boulder basados en mediciones GPS. 